\title{
False samples are not the same as blind controls
}

\section{Informal efforts to "test" a laboratory corrupt the data stream, where integrity is crucial.}

Sir - As director of the laboratory that analysed the samples for the National Lynx Survey sponsored by the United States Department of Agriculture's Forest Service (USFS), I am responding to the damaging yet unsubstantiated conclusions in your Opinion article "Lynch mob turns on lynx researchers" and News article "Fur flies over lynx survey's suspect samples" 2 about sampling for Canada lynx ( $\operatorname{Lyn} x$

canadensis).

My laboratory developed the protocol to distinguish among species of North American felids based on DNA analysis of hairs using known individuals across the geographical range of the target species, and comprehensively validated it using blind control tests both within our lab and at another lab. Our protocol was peer-reviewed and published ${ }^{3}$.

In practice, positive and negative controls are used with every sample set analysed. We have never misidentified a species when the identity of the sample was known, and we consistently detect lynx in areas where they are known to exist. In contrast to the implications of your Opinion article, therefore, the laboratory procedure to distinguish lynx based on hair samples is fully diagnostic and validated.

In 1999, the USFS implemented the National Lynx Survey to evaluate systematically the distribution of lynx on federal lands across 12 states; species identification based on DNA analysis was the final step. I was principal investigator in charge of DNA analysis, and Kevin McKelvey from the Rocky Mountain Research Station (USFS) was the principal investigator for the nationwide survey protocol. Field personnel (several hundred permanent and temporary federal and state employees) collected hairs from over $13,000 \mathrm{rub}^{\mathrm{pads}}{ }^{4}$ and sent them to the lab in labelled vials with accompanying data sheets. Because integrity of the data stream is particularly crucial in a study of such enormous scope and scale, the protocol was explicit, with comprehensive written instructions for all aspects of gathering, labelling and submitting samples.

For a field worker to arbitrarily decide "to test the lab" by labelling a hair from elsewhere as if it were a field-collected sample corrupts the integrity of the data and does not constitute a blind control. By analogy, a medical field worker who surreptitiously contaminates a blood sample to "test the rigour" of the serological protocol during a wide-scale survey to assay a disease is not conducting a blind control but is fabricating data that could lead to false conclusions about disease distribution.

I do not know the field personnel who submitted false samples and cannot assume anything about their motivation. But the vast majority of those participating in this unprecedented large-scale survey followed scientific protocol. The few who misrepresented data without notifying those who analyse and interpret the data did not, as your Opinion article suggested, merely implement blind controls.

Nobody contacted either me or the USFS research scientist in charge of the survey protocol about any concerns or told us about the plan to misrepresent data. In fact, we learned about the 1999 false samples only after the start of an internal USFS investigation.

I agree with your Opinion article that "Clean data are needed to prevent years of court battles over use of the forests" ${ }^{\text {. In }}$ this case, the methodology was peerreviewed, published, explicit, complete and followed by all except those few who intentionally mislabelled samples. The only potential smudge on the 'cleanliness' of data in the National Lynx Survey at this point comes from submission of samples not in compliance with scientific protocol.

Our Letter published on page 424 of this issue 5 is not part of the National Lynx survey; all 599 samples used in ref. 5 were collected from freezers and other known sources.

\section{Scott Mills}

Wildlife Biology Program, School of Forestry, University of Montana, Missoula,

Montana 59812, USA

1. Nature 415, 101 (2002).

2. Dalton, R. Nature 415,107 (2002).

3. Mills, L. S., Pilgrim, K. L., Schwartz, M. K. \& McKelvey, K. Conserv. Genet. 1, 285-288 (2000)

4. McDaniel, G. W., McKelvey, K. S., Squires, J. R. \& Ruggiero, L. F. Wildl. Soc. Bull. 28, 119-123 (2000). 5. Schwartz, M. K. et al. Nature 415, 424-426 (2002).

\section{Mislabelling muddies the forest-survey waters}

Sir - Calling people who object to deliberate mislabelling of samples a "lynch mob", as your Opinion article (Nature 415, $101 ; 2002)$ does, is like asserting that the collapse of the US energy company Enron was lamentable, and that those employees who lost their retirement savings deserve to be ashamed. It is half true (it is lamentable), but the implied criticism is completely misdirected.

Although conservatives in Congress and the media have indeed harvested a bounty of partisan hay from the misbegotten actions of some agency employees, several important issues remain unresolved.

It is not clear whether the field workers had any fraudulent intent or whether they intended the submitted lynx (Lynx canadensis) hairs to be controls in a test of the analytical lab. But it is clear that earlier samples submitted from the same geographical region to a different lab produced what were subsequently believed to be false positives - lynx hairs were reported from areas with no previous or subsequent evidence of lynx.

How the submission of blind positive controls could have provided a check on false positives is far from clear. More important, the laboratory supposedly being tested in the episode described in your article was in no way suspect in the earlier problems.

Any criticism should be levelled at the field workers who undertook a series of undocumented, informal steps that could be misinterpreted as fraud. Clearly, all would have benefited had they written a protocol, received documented approval, and notified the analytical lab that blind controls would be included in submitted samples.

\section{Steven W. Buskirk}

Department of Zoology and Physiology, University of Wyoming, Laramie, Wyoming 82071-3166, USA

\section{Regional network raises profile of local journals}

Sir - Researchers worldwide strive to publish in journals with high impact factors, but such journals are concentrated in developed countries, leaving journals in developing countries ranked at the bottom (see S. B. Vohora \& D. Vohora, Nature 412, $583 ; 2001)$. These journals are further imperilled by the decreasing level of support among local subscribers, who in the face of economic constraints may prefer to subscribe to journals with high impact factors.

Electronic publishing is bringing hope to these threatened journals. Citations increase when papers are freely available on 
the Internet (see S. Harnad, Nature 410, 1024-1025; 2001), and journals can increase their impact factors by publishing their contents electronically (see M. Curti, V. Pistotti, G. Gabutti \& C. Klersy, Haematologica 86, 1015-1020; 2001).

However, two shortcomings still stand out. First, online publication involves substantial costs (software development, hosting support, and so on), despite potential price cuts associated with the reduction of print editions. Second, free online access to local journals does not necessarily lead to increasing readership without a powerful means of dissemination. For these journals to be internationally recognized, regional networks with speedy access from search engines, portals and indexing services are required.

Such a network was launched in 1997: SciELO (Scientific Electronic Library Online: www.scielo.org) is a publicly funded initiative set up to promote cooperative, free electronic publishing of scientific journals from developing countries; the development of regional databases; and the implementation of indicators of scientific literature usage. It currently comprises 93 journals from Brazil, Chile and Cuba. We assessed its international impact by comparing the impact factors of journals before and after being incorporated in SciELO.

We found five Brazilian journals that had been indexed by ISI for at least five years and available in SciELO for at least two. The impact factors of these journals more than doubled (132.7\% increase, onetailed Wilcoxon signed ranks test, $P<0.02$ ) since their inclusion in SciELO.

This indicates that such networks not only foster the availability of scientific information on a regional scale, but also generate international impact which may entice researchers into publishing in the journals concerned. Those who fund and promote regionally coordinated networks for scientific electronic publishing can help developing countries to revitalize their local journals and enhance the international representation of locally generated knowledge.

Wladimir J. Alonso, Esteban FernándezJuricic

Department of Zoology, University of Oxford, Oxford OX1 3PS, UK

\section{Laboratories' gravy train has ground to a halt}

Sir - Your Opinion article "Time to halt the gravy train" (Nature 414, 829; 2001) questions the salary and benefits packages paid by international laboratories. It is entirely correct that these should be scrutinized at a time when cost efficiency is high on the agenda of most institutions, but some of the comparisons you make are oversimplified and misleading. The reality is that the gravy train ground to a halt long ago.

In comparing CERN (the European laboratory for particle physics) with DESY, Germany's main high-energy physics laboratory, which is in Hamburg, for example, you fail to take into account the significant cost-of-living differentials between Geneva and most other European locations.

As you rightly point out, CERN conducts regular tracking studies which compare our salary and benefits packages with industry and other institutions that compete for the scientific, engineering, IT and other skills we need. In this context, CERN is primarily a provider of research infrastructure, supporting a user community of more than 6,000 scientists — only 90 of our 2,700 current staff are engaged in fundamental research. We are far from topping the compensation table: indeed, in some skills areas we find it increasingly difficult to attract suitably qualified people away from their home markets, particularly in northern Europe.

As your leader implied, multinational institutions must be increasingly efficient in managing their resources and in being able to justify the investments they make. Maintaining the skills levels and human vitality of CERN is fundamental to being able to fulfil its mission as one of the world's leading scientific research establishments. In my view, the budgetary pressures we and many comparable institutions face are such that one of our biggest challenges will be to remain competitive in this respect.

Luciano Maiani

Director general, CERN, CH-1211, Geneva 23, Switzerland

\section{Tax-free pay lets funders evade responsibilities}

Sir - Although I agree with your Opinion article (Nature 414, 829; 2001) that the tax-free salary status of a few scientific researchers in Europe is an anachronism, I dispute your use of the phrase "gravy train”. Many life-science researchers are paid apparently generous tax-free stipends at some point in their careers, but these salaries are less generous after making reasonable provision for healthcare and retirement. Until Europe-wide agreements on these standard employment benefits have been reached, the alleged "gravy train" will continue.

You do not mention that government organizations such as the Max Planck

Society in Germany often pay postdoctoral researchers tax-free stipends that can hardly be described as generous between 17,850 euros (US\$15,344) and 22,000 euros a year, depending on the exact circumstances. After private healthcare (about 2,500 euros a year) and a basic pension (about 2,000 euros) have been deducted, this is not a great deal of money.

This practice should be stamped out. Surely scientists deserve the same pay and working conditions as other professionals? The era of the tax-free stipend is indeed over, but for very different reasons from the ones you suggest.

It is easy to print melodramatic headlines about the high tax-free salaries of a few physicists at CERN and to overlook the real problems faced by many more scientists around Europe. Tax-free stipends are simply a way that scientific institutions and funding bodies escape responsibilities to their employees.

\section{Francis Barr}

Department of Cell Biology, Max Planck

Institute of Biochemistry, Am Klopferspitz 18a, Martinsried 82152, Germany

\section{People, payments and positions at DESY}

Sir - In your Opinion article about benefits for scientists working abroad (Nature 414, 829; 2001) you state that only one-third of the scientists at DESY (Germany's high-energy accelerator centre) are German.

This is correct for the scientists who visit DESY to perform experiments as members of one of the four HERA collaborations or in the Synchrotron Radiation Laboratory HASYLAB. They are, however, not employed by DESY. Of the scientists on the DESY payroll, 75.4\% are German.

You also state that at DESY a young, unmarried graduate can expect to earn 18,500 euros (US\$16,346) a year. That figure is incorrect. The correct amount is 40,000 euros.

Petra Folkerts

DESY, Notkestrasse 85, D - 22607 Hamburg, Germany

The "young, unmarried graduate" positions discussed in the Opinion article referred to $\mathrm{PhD}$ students, who in Germany are usually paid a half-salary, which is typically 18,500 euros. People earning the full-time salary of around 40,000 euros cannot study for a PhD. Editor, Correspondence. 